\title{
Comparison of intracorporeal and extracorporeal urinary diversions after laparoscopic radical cystectomy in females with bladder cancer
}

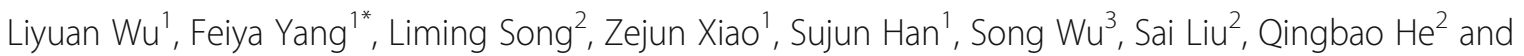
Nianzeng Xing ${ }^{1 *}$ (D)

\begin{abstract}
Purpose: To compare the peri-operative outcomes of females undergoing laparoscopic intracorporeal urinary diversions (ICUD) and extracorporeal urinary diversions (ECUD) after laparoscopic radical cystectomies (LRC).

Patients and methods: Thirty-eight females who underwent LRCs and urinary diversions from February 2008 to October 2018 were divided into two groups: the ECUD group (19 patients) and the ICUD group (19 patients). We retrospectively analysed the patients in terms of patients' demographics, peri-operative outcomes, and oncological follow-ups.
\end{abstract}

Results: There were significant differences in the mean operative times between ECUDs and ICUDs (364.6 vs. 297.1 min, $p=0.007$ ), transfusion rates ( $37 \%$ vs. $5 \%, p=0.042$ ), time to flatus ( 5 vs. 3 days, $p=0.020$ ), time to ambulation ( 2 vs. 1 days, $p=0.022$ ), and duration of postoperative hospital stays ( $22 \mathrm{vs} .13$ days, $p=0.002$ ). The mean lymph node yield was 12.9 in the ECUD group and 18.6 in the ICUD group $(p=0.140)$. Seven out of 19 patients (37\%) in the ECUD group and 6 out of 19 patients (32\%) in the ICUD group had positive lymph nodes $(p>0.9)$. Two out of 19 ECUD patients (11\%) and 4 of 19 ICUD patients (21\%) had positive surgical margins $(p=0.660)$. Although there were no differences in major complications at 30 days and in all complications at 90 days, the Clavien grade II complications were significantly different at 30 days (ECUD 8, ICUD 2; $p=0.026$ ). The mean follow-up times were 48.7 months (ECUD group) and 26.4 months (ICUD group). There were no statistically significant differences in estimated glomerular filtration rates postoperatively $(p=0.516)$. Seven patients had disease metastases (ECUD 2 out of 19, ICUD 5 out of 19; $p=0.405$ ) and 5 died (ECUD 3 out of 19, ICUD 2 out of 19; $p>0.9$ ).

Conclusions: ICUDs benefit females by having smaller incisions, faster recoveries, and decreased complication rates. Keywords: Female, Bladder cancer, Laparoscopic radical cystectomy, Surgical technique

\section{Introduction}

Primary bladder cancer is a serious worldwide health issue [1], with the male to female morbidity being 3:1 [2]. A radical cystectomy with pelvic lymph node dissection is considered the standard operative treatment for muscle-invasive bladder cancer (MIBC). Compared with

\footnotetext{
*Correspondence: yangfeiya2016@163.com; xingnianzeng@hotmail.com 'Department of Urology, National Cancer Center/National Clinical Research Center for Cancer/Cancer Hospital, Chinese Academy of Medical Sciences and Peking Union Medical College, No. 17, Panjiayuan South Li, Chaoyang district, Beijing 100021, People's Republic of China

Full list of author information is available at the end of the article
}

open surgery, a laparoscopic radical cystectomy (LRC) is a safe and feasible alternative with fewer complications, reliable pathology and oncologic efficacy, and faster recoveries [3]. Although extracorporeal urinary diversion (ECUD) after LRC has met the standard of care in medical centres for a long time, intracorporeal urinary diversion (ICUD) has gained popularity since the procedure was first described by Gill et al., in 2000 [4]. While traditional surgeries for female patients with MIBC involves anterior exenteration with dissection of the bladder, urethra, uterus, vagina, and both ovaries [5],

(C) The Author(s). 2019 Open Access This article is distributed under the terms of the Creative Commons Attribution 4.0 International License (http://creativecommons.org/licenses/by/4.0/), which permits unrestricted use, distribution, and 
there is significant controversy regarding which diversion is more beneficial for women after LRC. This article aims to compare ICUDs and ECUDs following LRCs in female patients by analysing their demographics, perioperative outcomes, and oncological follow-ups.

\section{Materials and methods Patients}

From February 2008 to October 2018, 38 female patients with bladder cancer received LRCs with urinary diversions, of whom 19 patients underwent LRCs with ECUDs and 19 underwent LRCs with ICUDs. All operations were performed by an experienced surgeon in a single centre. Indications included muscle invasive bladder cancer, T1G3 and recurrent superficial bladder cancer, or extensive non-muscle-invasive bladder cancer (NMIBC) that could not be controlled by transurethral resection bladder cancer and intravesical treatments. All patients received pelvic magnetic resonance imaging or enhanced computed tomography examinations before their surgeries. No distant metastases were identified on bone scans, chest X-rays, and sonographies. The Clavien-Dindo classification was used to assess postoperative complications [6].

\section{Surgical technique}

Under intravenous anaesthesia, patients were placed in lithotomy positions. We used a five-port transperitoneal approach. The first $10-\mathrm{mm}$ trocar (for the camera) was placed at the $2-4 \mathrm{~cm}$ upper level of the umbilicus. Two $12-\mathrm{mm}$ trocars were placed at the bilateral external rectus line, and two $5-\mathrm{mm}$ trocars were placed at the $3-\mathrm{cm}$ interior bilateral anterior superior iliac spines, at the same level as the first trocar.

\section{Anterior pelvic exenteration}

Laparoscopic dissection began with generous mobilisation of the suspensory ligament of the ovary. The suspensory ligaments were transected with Ligasure $^{\mathrm{TM}}$ (Valleylab, USA). The bilateral ureters were then divided at the common iliac artery bifurcation and the umbilical arteries were dissected using Hem-O-Lok clips. The broad ligaments of the uterus were disassociated along the pelvic wall and the cardinal ligament of the uterus was cut off. The fornix of the vagina was incised transversely until reaching the posterior urethra, after lifting the uterus and bilateral attachments using forceps. Subsequently, the endopelvic fascia was dissected at both sides, and the dorsal vein complex (DVC) was ligated with 2-0 V-Loc sutures to avoid using instruments that caused heat damage, thereby preserving sexual function. The anterior urethra was separated along the bladder neck and removed using scissors in a 'cone shape' to protect the internal urethral sphincter. Hem-O-Lok clips were used to close the urethra. The ureters were also closed using Hem-O-Lok clips (two towards the bladder and one towards the proximal end) and were then transected down the middle using scissors. Frozen section examinations of the ends of the urethra and the distal ureter were subsequently performed. The bladder and the female reproductive organs were placed into an EndoCatch bag and were removed together through the vagina, which was later closed with a running suture.

\section{Pelvic lymph node dissection}

The external iliac artery sheath was cut from the iliac artery branch to the distal end. Dissections of the internal iliac lymph nodes, pre-sacral lymph nodes, obturator fossa lymph nodes, and external iliac lymph nodes were then performed. Distinct area lymph nodes were marked and removed for pathological examination.

\section{Urinary diversion}

For ECUDs, the ileum was extracted through the 10-15$\mathrm{cm}$ incision below the umbilicus. For ileal conduits [7], a 15 -cm-long ileal segment was isolated about $20 \mathrm{~cm}$ proximally to the ileocaecum; for orthotopic ileal neobladders [8], we choose a $60-\mathrm{cm}$-long ileal segment. The ileal segments were detubularised along their anti-mesenteric border to form a new reservoir outside the abdominal cavity. Before the suturing of the new reservoir was complete, both ureters were spatulated for approximately $3 \mathrm{~cm}$ and Mono-J ureteric stents (6F) were inserted in both ureters. The ureters were then anastomosed with the proximal end of reservoir end-toend. Finally, a circular disc of skin was excised at the right 12-mm port, and the outflow limb was fixed with fascia and skin. For ICUDs, the above urinary diversion was completed in the abdominal cavity. Nineteen patients underwent ECUD and 19 patients underwent ICUD.

\section{Statistical analysis}

Continuous data were analysed using mean values with standard deviations and compared by Student's $t$ tests. Categorical data were analysed using medians and ranges and compared with the test. $p$ values $<0.05$ were considered statistically significant. Kaplan-Meier analysis was used to calculate survival probabilities. All statistics were performed using SPSS v. 21 (IBM Corp, Armonk, NY, USA).

\section{Results}

There were no significant differences in baseline characteristics, body mass indices, Charlson comorbidity indices, American Society of Anaesthesiologists scores, pre-creatinine, and pre-operative stage distributions, etc. (Table 1). 
Table 1 Patients demographics

\begin{tabular}{|c|c|c|c|}
\hline Variables & Extracorporeal & Intracorporeal & $P$ \\
\hline Patients, $n$ & 19 & 19 & \\
\hline Age (mean $\pm S D)$, year & $62.5 \pm 8.54$ & $67.9 \pm 11.40$ & 0.173 \\
\hline BMI (mean $\pm S D), \mathrm{kg} / \mathrm{m}^{2}$ & $25.5 \pm 2.97$ & $25.3 \pm 2.74$ & 0.867 \\
\hline Charlson comorbidity index (median [range]) & $4[2-9]$ & $3[2-5]$ & 0.548 \\
\hline ASA score, $n$ & & & $>0.9$ \\
\hline $1-2$ & 17 (89\%) & 17 (89\%) & \\
\hline 3 & $2(11 \%)$ & $2(11 \%)$ & \\
\hline Neoadjuvant chemotherapy, n (\%) & $4(21 \%)$ & $3(16 \%)$ & $>0.9$ \\
\hline Pre-eGFR $($ mean $\pm \mathrm{SD}), \mathrm{mL} /\left(\min \cdot 1.73 \mathrm{~m}^{2}\right)$ & $86.3 \pm 28.87$ & $85.9 \pm 34.57$ & 0.972 \\
\hline Pre-Hb (mean $\pm \mathrm{SD}), \mathrm{g} / \mathrm{L}$ & $116.9 \pm 16.81$ & $118.6 \pm 13.94$ & 0.765 \\
\hline Preoperative diagnosis, $n$ & & & $>0.9$ \\
\hline NMIBC & $7(37 \%)$ & $8(42 \%)$ & \\
\hline MIBC & $12(63 \%)$ & $11(58 \%)$ & \\
\hline Clinical stage & & & 0.720 \\
\hline $\mathrm{T}_{\mathrm{a}} / \mathrm{T}_{1} / \mathrm{T}_{\mathrm{is}}$ & $7(37 \%)$ & $8(42 \%)$ & \\
\hline $\mathrm{T}_{2}$ & $9(47 \%)$ & $5(26 \%)$ & \\
\hline$T_{3}$ & $3(16 \%)$ & $5(26 \%)$ & \\
\hline $\mathrm{T}_{4}$ & $0(0 \%)$ & $1(5 \%)$ & \\
\hline Clinical stage & & & 0.405 \\
\hline$N_{0}$ & 17 (89\%) & 14 (74\%) & \\
\hline $\mathrm{N}_{1}$ & $2(11 \%)$ & $5(26 \%)$ & \\
\hline
\end{tabular}

$B M I$ body mass index, ASA American Society of Anesthesiologists, Pre-eGFR pre-estimated glomerular filtration rate, MIBC muscle-invasive bladder cancer, NMIBC non-muscle-invasive bladder cancer

A total of 38 patients underwent LRCs with ICUDs or ECUDs: 19 had ECUDs (11 conduits and 8 neobladders), and 19 had ICUDs (13 conduits and 6 neobladders) (Table 2). There were significant differences in ECUDs and ICUDs for mean operative times $(364.6 \pm 72.30 \mathrm{vs}$. $297.1 \pm 48.24 \mathrm{~min}, p=0.007)$, transfusion rates $(37 \% \mathrm{vs}$. $5 \%, p=0.042$ ), time to flatus (5 vs. 3 days, $p=0.020$ ), time to ambulation ( 2 vs. 1 days, $p=0.022$ ), and duration of postoperative hospital stays (22 vs. 13 days, $p=0.002$ ). However, there were no statistical differences in estimated blood loss (EBL) (393.1 \pm 387.46 vs. $267.0 \pm$ 205.69, $p=0.252$ ), intensive care unit (ICU) stay rates post-surgery $(10 \%$ vs. $5 \%, p>0.9)$, onset of liquid diets $(5$ vs. 4 days, $p=0.087)$, and length of time before JacksonPratt drains were removed (14 vs. 10 days, $p=0.549)$. Overall, there were no significant differences in pathologic stages $(p=0.527)$. Mean lymph node yield was 12.9 in the ECUD group and 18.6 in the ICUD group $(p=0.140)$. Seven out of 19 patients $(37 \%)$ in the ECUD group and 6 out of 19 patients (32\%) in the ICUD group had positive lymph nodes $(p>0.9)$. Two out of 19 patients in the ECUD group (11\%) and 4 of 19 patients in the ICUD group (21\%) had positive surgical margins $(p=0.660)$.
For ileal conduits, there were statistical differences between ECUD and ICUD transfusion rates (46\% vs. $7.7 \%$, $p=0.048$ ); however, there were no statistical differences in the mean operative times $(330.0 \pm 53.67$ vs. $288.5 \pm$ $45.99 \mathrm{~min}, p=0.101)$, EBLs $(600.0 \pm 485.80$ vs. $242.7 \pm$ $178.75, p=0.134)$, postoperative ICU stay rates (18\% vs. $8 \%, p=0.435$ ), time to flatus ( 4 vs. 3 days, $p=0.099$ ), onset of liquid diets ( 5 vs. 4 days, $p=0.169$ ), time to ambulation ( 1 vs. 1 days, $p=0.052$ ), time before removal of the Jackson-Pratt drains (12 vs. 8 days, $p=0.758$ ), and duration of postoperative hospital stays (15 vs. 14 days, $p=0.234$ ) (Table 3). For neobladder reconstructions, there were statistical differences in the duration of postoperative hospital stays (23 days [ECUD] vs. 10 days [ICUD], $p=0.039)$. There were no statistical differences in any other categories (Table 4).

Overall, there were no statistical differences in the rates of minor and major 30 days or 90 days complications (Table 5). In the ECUD group, 10 patients (52\%) presented with at least one complication within 30 days, and 9 of these patients (47\%) had minor complications (grades 1-2). Similarly, 6 patients (37\%) in the ICUD group presented with at least one complication and 1 (5\%) had a Clavien grade V complication who later died 
Table 2 Surgical outcomes for female patients

\begin{tabular}{|c|c|c|c|}
\hline Variables & Extracorporeal $(n=19)$ & Intracorporeal $(n=19)$ & $P$ \\
\hline Diversion type, $n(\%)$ & & & 0.737 \\
\hline Ileal conduit & $11(58 \%)$ & $13(68 \%)$ & \\
\hline Neobladder & $8(42 \%)$ & $6(32 \%)$ & \\
\hline Operative time (mean \pm SD), $\min$ & $364.6 \pm 72.30$ & $297.1 \pm 48.24$ & 0.007 \\
\hline $\mathrm{EBL}($ mean $\pm \mathrm{SD}), \mathrm{mL}$ & $393.1 \pm 387.46$ & $267.0 \pm 205.69$ & 0.252 \\
\hline Transfusion, $n(\%)$ & $7(37 \%)$ & $1(5 \%)$ & 0.042 \\
\hline ICU stay after surgery, n (\%) & $2(10 \%)$ & $1(5 \%)$ & $>0.9$ \\
\hline Time to flatus (median [range]), days & $5[3-9]$ & $3[1-6]$ & 0.020 \\
\hline Time to intake of liquid diet (median [range]), days & $5[4-8]$ & $4[2-18]$ & 0.087 \\
\hline Time to ambulation (median [range]), days & $2[0-5]$ & $1[1-3]$ & 0.022 \\
\hline Time to remove Jackson-Pratt drain (median [range]), days & $14[5-23]$ & $10[4-30]$ & 0.549 \\
\hline Length of hospital stay after surgery (median [range]), days & $22[14-45]$ & $13[7-22]$ & 0.002 \\
\hline Final pathologic stage & & & 0.527 \\
\hline $\mathrm{T}_{\mathrm{a}} / \mathrm{T}_{1} / \mathrm{T}_{\text {is }}$ & $10(53 \%)$ & $9(47 \%)$ & \\
\hline $\mathrm{T}_{2}$ & $6(32 \%)$ & $4(21 \%)$ & \\
\hline $\mathrm{T}_{3}$ & $2(10 \%)$ & $5(26 \%)$ & \\
\hline $\mathrm{T}_{4}$ & $1(5 \%)$ & $1(5 \%)$ & \\
\hline Pathologic stage & & & 0.631 \\
\hline $\mathrm{N}_{0}$ & $13(68 \%)$ & $13(68 \%)$ & \\
\hline $\mathrm{N}_{1}$ & $3(16 \%)$ & $0(0 \%)$ & \\
\hline $\mathrm{N}_{2}$ & $3(16 \%)$ & $3(16 \%)$ & \\
\hline $\mathrm{N}_{3}$ & $0(0 \%)$ & $3(16 \%)$ & \\
\hline Lymph node yield (mean \pm SD), $n$ & $12.9 \pm 7.81$ & $18.6 \pm 11.51$ & 0.140 \\
\hline Lymph node positive patients, $n(\%)$ & $7(37 \%)$ & $6(32 \%)$ & $>0.9$ \\
\hline Positive surgical margin, $n$ (\%) & $2(11 \%)$ & $4(21 \%)$ & 0.660 \\
\hline
\end{tabular}

EBL estimated blood loss

Table 3 Surgical outcomes for ileal conduit

\begin{tabular}{llll}
\hline Variables & Extracorporeal $(n=11)$ & Intracorporeal $(n=13)$ & $P$ \\
\hline Operative time (mean \pm SD), min & $330.0 \pm 53.67$ & $288.5 \pm 45.99$ & 0.101 \\
EBL (mean \pm SD), $\mathrm{mL}$ & $600.0 \pm 485.80$ & $242.7 \pm 178.75$ & $1(7.7 \%)$ \\
Transfusion, $n(\%)$ & $5(46 \%)$ & $1(8 \%)$ & 0.134 \\
ICU stay after surgery, $n(\%)$ & $2(18 \%)$ & $3[1-6]$ & 0.048 \\
Time to flatus (median [range]), days & $4[3-7]$ & $4[2-18]$ & 0.435 \\
Time to intake of liquid diet (median [range]), days & $5[4-8]$ & $1[1-3]$ & 0.099 \\
Time to ambulation (median [range]), days & $1[0-2]$ & $8[4-30]$ & 0.169 \\
Time to remove Jackson-Pratt drain (median [range]), days & $12[7-17]$ & $14[7-22]$ & 0.052 \\
Length of hospital stay after surgery (median [range]), days & $15[14-23]$ & $20.2 \pm 11.08$ & 0.758 \\
Lymph node yield (mean \pm SD), $n$ & $9.8 \pm 10.15$ & $5(39 \%)$ & 0.234 \\
Lymph node positive patients, $n$ (\%) & $5(46 \%)$ & $3(23 \%)$ & 0.070 \\
Positive surgical margin, $n(\%)$ & $2(18 \%)$ & 0.527 \\
\hline
\end{tabular}


Table 4 Surgical outcomes for neobladder

\begin{tabular}{llll}
\hline Variables & Extracorporeal $(n=8)$ & Intracorporeal $(n=6)$ & $P$ \\
\hline Operative time (mean \pm SD), min & $394.3 \pm 76.35$ & $352.5 \pm 10.61$ & $425.0 \pm 388.91$ \\
EBL (mean \pm SD), $\mathrm{mL}$ & $230.0 \pm 158.01$ & 0.486 \\
Transfusion, $n$ (\%) & $2(25 \%)$ & $0(0 \%)$ & 0.606 \\
ICU stay after surgery, $n(\%)$ & $0(0 \%)$ & $2[3-5]$ & 4.308 \\
Time to flatus (median [range]), days & $5[3-9]$ & $4[3-5]$ & $2[2-3]$ \\
Time to intake of liquid diet (median [range]), days & $5[4-6]$ & $18[8-28]$ & 0.361 \\
Time to ambulation (median [range]), days & $2[1-5]$ & $10[7-15]$ & 0.280 \\
Time to remove Jackson-Pratt drain (median [range]), days & $16[5-23]$ & $8.5 \pm 12.02$ & 0.190 \\
Length of hospital stay after surgery (median [range]), days & $23[20-45]$ & $1(17 \%)$ & 0.769 \\
Lymph node yield (mean \pm SD), $n$ & $15.4 \pm 4.39$ & $1(17 \%)$ & 0.039 \\
Lymph node positive patients, $n(\%)$ & $2(25 \%)$ & 0.562 \\
Positive surgical margin, $n$ (\%) & $0(0 \%)$ & $6(75 \%)$ & $3(50 \%)$ \\
Complete continence in the daytime, $n(\%)$ & $6(75 \%)$ & 0.615 \\
Complete continence at night, $n(\%)$ & & 0.429 \\
\hline
\end{tabular}

due to multiple organ failure resulting from a myocardial infarction within 30 days. Interestingly, there were significant differences at Clavien grade II (ECUD 8, ICUD $2 ; p=0.026$ ) within 30 days. The most common complications in the ECUD group were haematological diseases, while infections were the most common in the ICUD group (Tables 6 and 7).

\section{Follow-up data}

The mean follow-up times were 48.7 and 26.4 months for the ECUD and ICUD groups, respectively. There were no statistically significant differences in the estimated glomerular filtration rate postoperatively $(p=0.516)$. Seven patients had disease metastases (ECUD 2 out of 19, ICUD 5 out of $19 ; p=0.405$ ) and 5 died (ECUD 3 out of 19, ICUD 2 out of 19; $p>0.9$ ). There was no recurrence. There was no statistical differences in oncologic outcomes (recurrence, metastasis, or overall mortality) (Table 5) or KaplanMeier analyses between the two groups (Fig. 1).

\section{Discussion}

An LRC with an ICUD is technically challenging and requires extensive operative experience. The procedure has seldomly been reported since the first two cases reported by Gill et al. [9]. Recently, with the popularisation and improvement of laparoscopic techniques, LRCs and urinary diversions have become more popular in female patients, although females, with their distinctive pelvic anatomies, have many surgical differences in radical cystectomies compared with males. While limited reports on laparoscopic radical cystectomies in females have begun to emerge, this is the first paper comparing intracorporeal and extracorporeal urinary diversions after LRCs in women. These operations were performed entirely by one surgeon in a single centre.

Table 5 Follow-up data

\begin{tabular}{llll}
\hline Variables & Extracorporeal $(n=19)$ & Intracorporeal $(n=19)$ & $P$ \\
\hline Follow-up time (mean \pm SD), months & $48.7 \pm 30.58$ & $26.4 \pm 8.29$ & 0.329 \\
Post- eGFR (mean \pm SD), $\mathrm{mL} /\left(\min \cdot 1.73 \mathrm{~m}^{2}\right)$ & $79.0 \pm 19.95$ & $86.2 \pm 34.7$ & 0.516 \\
30-day complication rates, $n(\%)$ & & & 0.227 \\
$\quad$ Minor (I-II) & $9(47 \%)$ & $5(32 \%)$ & 0.313 \\
$\quad$ Major (III-V) & $1(5 \%)$ & $1(5 \%)$ & $>0.9$ \\
90-day complication rates, $n(\%)$ & & & 0.142 \\
$\quad$ Minor (I-II) & $1(5 \%)$ & $2(11 \%)$ & $>0.9$ \\
$\quad$ Major (III-V) & $0(0 \%)$ & $2(11 \%)$ & 0.486 \\
Metastasis, $n(\%)$ & $2(11 \%)$ & $5(26 \%)$ & 0.405 \\
Overall survival, $n(\%)$ & $16(84 \%)$ & $17(89 \%)$ & $>0.9$ \\
\hline
\end{tabular}

Post-eGFR post-estimated glomerular filtration rate 
Table 6 Complications classified by Clavien grade

\begin{tabular}{|c|c|c|c|c|c|c|}
\hline \multirow{2}{*}{$\begin{array}{l}\text { Clavien } \\
\text { grade }\end{array}$} & \multicolumn{3}{|c|}{30 days } & \multicolumn{3}{|c|}{90 days } \\
\hline & ECUD & ICUD & $P$ & ECUD & ICUD & $P$ \\
\hline 1 & 1 & 3 & 0.302 & 0 & 1 & $>0.9$ \\
\hline 2 & 8 & 2 & 0.026 & 1 & 1 & $>0.9$ \\
\hline 3 & 1 & 0 & $>0.9$ & 0 & 1 & $>0.9$ \\
\hline 4 & 0 & 0 & & 0 & 0 & \\
\hline 5 & 0 & 1 & $>0.9$ & 0 & 1 & $>0.9$ \\
\hline
\end{tabular}

Hussein et al. [10] retrospectively evaluated the outcomes of robot-assisted radical cystectomies with ICUDs $(n=1094)$ and ECUDs $(n=1031)$ from 26 institutions since 2005. Patients having ICUDs showed shorter operative times ( $357 \mathrm{~min}$ vs. $400 \mathrm{~min}, p<0.001$ ), less blood loss $(300 \mathrm{~mL}$ vs. $350 \mathrm{~mL}, p<0.001)$, and fewer received blood transfusions (5\% vs. $13 \%, p<0.001)$, suggesting that the complete laparoscopic approach was technically more efficient. Similarly, in our study, the mean operative time between the ECUD and ICUD groups (364.6 \pm 72.30 vs. $297.1 \pm 48.24 \mathrm{~min}, p=0.007)$, transfusion rates ( $37 \%$ vs. $5 \%, p=0.042$ ), and EBLs $(400.8 \mathrm{~mL}$ vs. 267.0 $\mathrm{mL}, p=0.252$ ) showed advantages for ICUDs to some extent. The time to flatus ( 3 days, $p=0.020$ ), ambulation ( 1 days, $p=0.022)$, and durations of hospital stays (13 days, $p=0.002$ ) postoperatively in the ICUD group were shorter than in the ECUD group. It was suggested that ICUDs were also associated with quicker recoveries. This may be partially due to decreased intestinal exposures, shorter operative times, and smaller EBLs. Additionally, enhanced recovery programmes, which we began in 2014 [11], were identified as an important part of postoperative treatments for radical cystectomies [12] that may have resulted in faster patient recoveries in the ICUD group in our study.
Ahmed et al. reported on 935 patients in 18 international centres who had undergone ICUDs or ECUDs following robot-assisted radical cystectomies resulting in $43 \%$ in the ECUD group and 35\% in the ICUD group having complications within 30 days of surgery $(p=0.07)$. The 90 -day complication rate was not significant between the two groups (ICUD $41 \%$ vs. ECUD $49 \%, p=0.055$ ) [13]. In our study, Clavien grade II complications were significantly different at 30 days (ECUD 8, ICUD 2; $p=0.026$ ) potentially indicating that the ICUD group was associated with decreased complication rates. Azzouni et al. studied 81 patients after robot-assisted intracorporeal ileal conduits (overall complication rate, $81 \%$ ) and reported postoperative complications in the first 90 days, 15 patients of whom had high-grade complications (15\%). A total of 164 complications were recorded with the most common complication being urinary tract infections [14]. Infections were also more common in the ICUD group in our study, with four patients having postoperative complications within 90 days $(22 \%)$ and two patients having high-grade complications (11\%). This indicates that our surgical technique could have had some benefits in terms of reducing postoperative complications and that robot-assisted intracorporeal ileal conduits are not better than laparoscopies for postoperative complications within 90 days.

In this group, orthotopic ileal neobladder reconstructions were performed in 14 cases, all of whom could urinate on their own 12 months postoperatively without catheterisation. The complete daytime continence rate was $88.9 \%$ and $77.8 \%$ at night. Orthotopic ileal neobladder reconstructions can maximally imitate the natural urination function and significantly improve the patients' psychological acceptance of surgery. To some extent, it

Table 7 Complications by system

\begin{tabular}{|c|c|c|c|c|}
\hline & \multicolumn{2}{|l|}{ ECUD } & \multicolumn{2}{|l|}{ ICUD } \\
\hline & $0-30$ days & 30-90 days & $0-30$ days & 30-90 days \\
\hline \multicolumn{5}{|l|}{ Haematological $(n=11)$} \\
\hline Hypoproteinemia & 8 & 1 & 0 & 0 \\
\hline Great saphenous varicosity & 1 & 0 & 0 & 0 \\
\hline Deep venous thrombosis & 0 & 0 & 1 & 0 \\
\hline \multicolumn{5}{|l|}{ Infectious ( $n=5$ ) } \\
\hline Wound infection & 0 & 0 & 1 & 0 \\
\hline Pneumonia & 1 & 0 & 0 & 1 \\
\hline Vaginitis & 0 & 0 & 2 & 0 \\
\hline \multicolumn{5}{|l|}{ Gastrointestinal $(n=3)$} \\
\hline Ileus & 0 & 0 & 1 & 2 \\
\hline Multiple organ failure $(n=1)$ & 0 & 0 & 1 & 0 \\
\hline Pulmonary metastasis $(n=1)$ & 0 & 0 & 0 & 1 \\
\hline
\end{tabular}




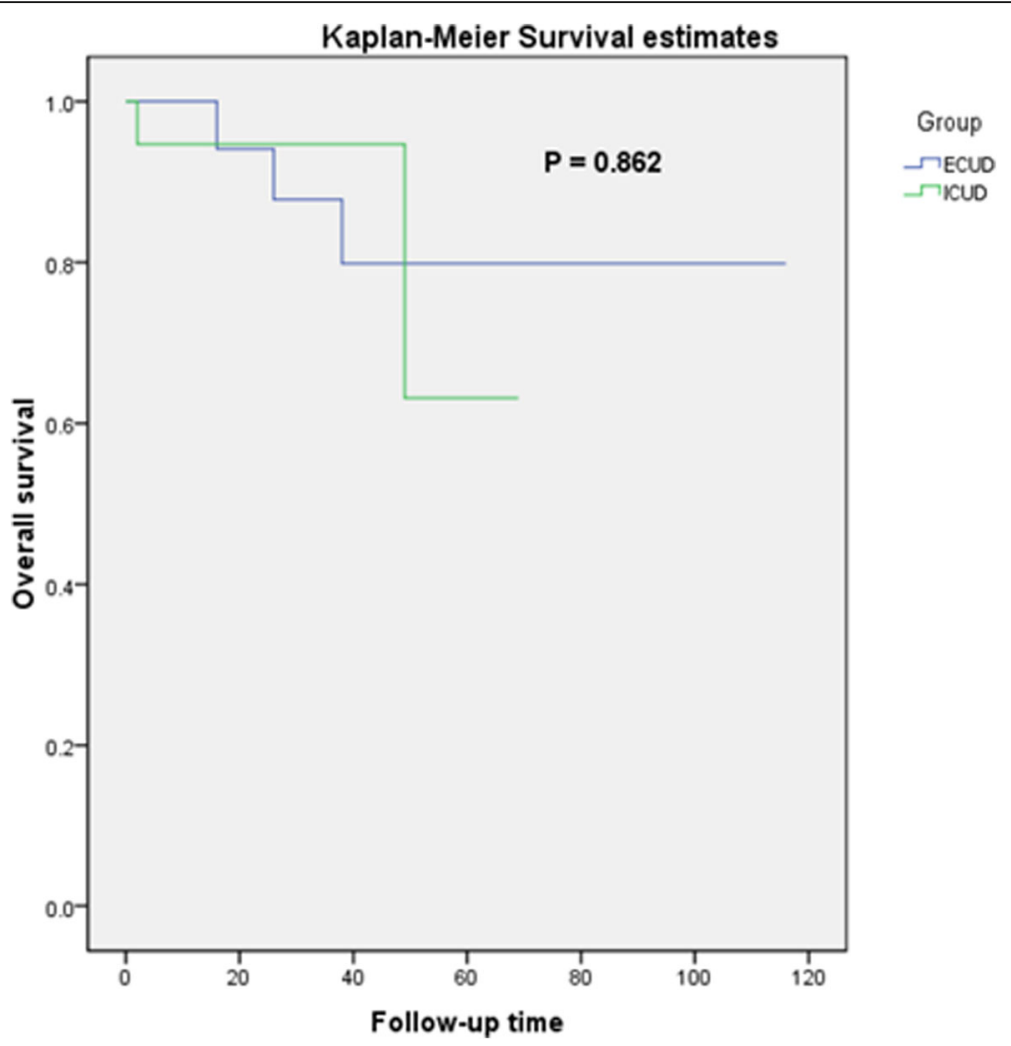

Fig. 1 Kaplan-Meier survival estimates between the ECUD and ICUD groups. Log-rank test $p=0.862$

might ameliorate the postoperative quality of life. Our experience regarding orthotopic ileal neobladder reconstructions are as follows: (1) the procedure should comply with the principles of tumour resection, and the urethral autonomic nerve should be preserved to the greatest extent; (2) the bladder neck should be separated close to the bladder wall; and (3) the bilateral tissue of the anterior vaginal wall should be preserved. Overall, the patients were satisfied with their postoperative urinary continence based on the follow-up results.

This study also had several limitations. First, the sample size was relatively small; thus, it was difficult to arrive at a definitive conclusion. Secondly, this was a cohort study and not a randomised trial; therefore, there were selection biases and limited information.

\section{Conclusion}

ICUD after LRC in women is technically feasible. Understanding the female pelvic anatomy and mastering the techniques are helpful to optimise surgical procedures and greatly decrease complications. Compared with ECUDs, ICUDs are potentially beneficial due to smaller incisions, faster recoveries, and decreased complication rates.

\section{Abbreviations}

EBL: Estimated blood loss; ECUD: Extracorporeal urinary diversion; ICUD: Intracorporeal urinary diversion; LRC: Laparoscopic radical cystectomy; MIBC: Muscle-invasive bladder cancer; NMIBC: Non-muscle-invasive bladder cancer

\section{Acknowledgements \\ Not applicable}

\section{Authors' contributions}

LYW contributed to the project development, data collection and management, data analysis, and manuscript writing. FYY contributed to the project development and manuscript editing. LYS, ZJX, SJH, and SW

contributed to the manuscript editing. SL and QBH contributed to the data collection and management. NZX contributed to the project development, data collection and management, and manuscript editing. All authors' read and approved the final manuscript.

\section{Funding}

This study was funded by the Capital Science and Technology Leading Talent Project supported by Beijing Municipal Science and Technology Commission (grant number: Z181100006318007).

\section{Availability of data and materials}

The datasets supporting the conclusions of this article are included within the article.

\section{Ethics approval and consent to participate}

The study was approved by the institutional research committee and was performed in accordance with the ethical standards as laid down in the 1964 Declaration of Helsinki and its later amendments or comparable ethical standards. All the patients in this study provided written informed consent. 


\section{Consent for publication}

Written informed consent was obtained from the patient for publication.

\section{Competing interests}

The authors declare that they have no competing interests.

\section{Author details}

'Department of Urology, National Cancer Center/National Clinical Research Center for Cancer/Cancer Hospital, Chinese Academy of Medical Sciences and Peking Union Medical College, No. 17, Panjiayuan South Li, Chaoyang district, Beijing 100021, People's Republic of China. ${ }^{2}$ Department of Urology, Beijing Chaoyang Hospital, Capital Medical University, No.8 Gongren Tiyuchang Nanlu, Chaoyang district, Beijing 100020, People's Republic of China. ${ }^{3}$ Department of Urology, Shenzhen Second People's Hospital, The First Affiliated Hospital of Shenzhen University, No. 3002, Sungang West Road, Futian District, Shenzhen 518000, People's Republic of China.

Received: 20 March 2019 Accepted: 25 July 2019

Published online: 12 September 2019

\section{References}

1. Chavan S, Bray F, Lortet-Tieulent J, Goodman M, Jemal A. International variations in bladder cancer incidence and mortality. Eur Urol. 2014;66:59-73.

2. Siegel RL, Miller KD, Fedewa SA, et al. Colorectal cancer statistics, 2017. CA Cancer J Clin. 2017;67:177-93.

3. Tang K, Li H, Xia D, et al. Laparoscopic versus open radical cystectomy in bladder cancer: a systematic review and meta-analysis of comparative studies. PLoS One. 2014;9:e95667.

4. Moinzadeh A, Gill IS, Desai M, et al. Laparoscopic radical cystectomy in the female. J Urol. 2005;173:1912-7.

5. Marshall FF, Treiger BF. Radical cystectomy (anterior exenteration) in the female patient. Urol Clin North Am. 1991;18:765-75.

6. Dindo D, Demartines N, Clavien PA. Classification of surgical complications: a new proposal with evaluation in a cohort of 6336 patients and results of a survey. Ann Surg. 2004;240:205-13.

7. Wang MS, He QB, Yang FY, Ping H, Xing NZ. A retrospective study comparing surgical and early oncological outcomes between intracorporeal and extracorporeal ileal conduit after laparoscopic radical cystectomy from a single center. Chin Med J (Engl). 2018;131:784-9.

8. Xing NZ, Kang N, Song LM, et al. Laparoscopic radical cystectomy with novel orthotopic neobladder with bilateral isoperistaltic afferent limbs: initial experience. Int Braz J Urol. 2017;43:57-66.

9. Gill IS, Fergany A, Klein EA, et al. Laparoscopic radical cystoprostatectomy with ileal conduit performed completely intracorporeally: the initial 2 cases. Urology. 2000;56:26-29; discussion 29-30.

10. Hussein AA, May PR, Jing Z, et al. Outcomes of intracorporeal urinary diversion after robot-assisted radical cystectomy: results from the international robotic cystectomy consortium. J Urol. 2018:199:1302-11.

11. Arumainayagam N, McGrath J, Jefferson KP, Gillatt DA. Introduction of an enhanced recovery protocol for radical cystectomy. BJU Int. 2008:101:698-701.

12. Smith J, Pruthi RS, McGrath J. Enhanced recovery programmes for patients undergoing radical cystectomy. Nat Rev Urol. 2014;11:437-44.

13. Ahmed K, Khan SA, Hayn MH, et al. Analysis of intracorporeal compared with extracorporeal urinary diversion after robot-assisted radical cystectomy: results from the International Robotic Cystectomy Consortium. Eur Urol. 2014;65:340-7

14. Azzouni FS, Din R, Rehman S, et al. The first 100 consecutive, robot-assisted, intracorporeal ileal conduits: evolution of technique and 90-day outcomes. Eur Urol. 2013:63:637-43.

\section{Publisher's Note}

Springer Nature remains neutral with regard to jurisdictional claims in published maps and institutional affiliations.

Ready to submit your research? Choose BMC and benefit from:

- fast, convenient online submission

- thorough peer review by experienced researchers in your field

- rapid publication on acceptance

- support for research data, including large and complex data types

- gold Open Access which fosters wider collaboration and increased citations

- maximum visibility for your research: over $100 \mathrm{M}$ website views per year

At $\mathrm{BMC}$, research is always in progress.

Learn more biomedcentral.com/submissions 